\title{
Portfolio Optimization Problem with Delay under Cox-Ingersoll-Ross Model
}

\author{
Chunxiang A, Yi Shao \\ School of Mathematics and Statistics, Zhaoqing University, Zhaoqing, China \\ Email: acxgmaria@zuq.edu.cn, mathsyishao@126.com
}

How to cite this paper: A, C.X. and Shao, Y. (2017) Portfolio Optimization Problem with Delay under Cox-Ingersoll-Ross Model. Journal of Mathematical Finance, 7, 699-717.

https://doi.org/10.4236/jmf.2017.73037

Received: June 2, 2017

Accepted: July 28, 2017

Published: July 31, 2017

Copyright $\odot 2017$ by authors and Scientific Research Publishing Inc. This work is licensed under the Creative Commons Attribution International License (CC BY 4.0).

http://creativecommons.org/licenses/by/4.0/

\begin{abstract}
This paper considers a portfolio optimization problem with delay. The finance market is consisted of one risk-free asset and one risk asset which price process is modeled by Cox-Ingersoll-Ross stochastic volatility model. In addition, considering the history information related to investment performance, the dynamic of wealth is modeled by stochastic delay differential equation. The investor's objective is to maximize her expected utility for a linear combination of the terminal wealth and the average performance. By applying stochastic dynamic programming approach, we provide the corresponding Hamilton-Jacobin-Bellman equation and verification theorem, and the closedform expressions of optimal strategy and optimal value function for CRRA utility are derived. Finally, a numerical example is provided to show our results.
\end{abstract}

\section{Keywords}

Portfolio, Stochastic Delay Differential Equation, Stochastic Volatility, Hamilton-Jacobin-Bellman Equation

\section{Introduction}

In this paper, we consider a portfolio optimization problem with delay, in which the Cox-Ingersoll-Ross (CIR) stochastic volatility model is adopted to describe a non-constant volatility of the risky asset. The phenomenon of frowns and smiles for the volatility of stock price cannot be explained within constant volatility models, stochastic volatility (SV) is recognized recently as an important feature for asset price models. There is much literature embedding SV in assets' returns. For example, Hull and White [1] assume that the volatility follows log-normal process; Scott [2] and Stein \& Stein [3] assume that the volatility follows Omstein-Uhlenbeck (OU) process; Heston [4] and Ball \& Roma [5] introduce CIR process to describe stochastic volatility. Furthermore, many scholars study 
the optimal investment and/or consumption problems under the SV models. For instance, Chacko and Viceira [6], Fleming and Hernndez-Hernndez [7], Liu [8] and Zariphopoulou [9] consider an optimal investment and consumption problems under SV models, and they derive explicitly the optimal strategies and optimal value functions in some situations by applying Hamilton-JacobinBellman (HJB) technique; Kraft [10], Taksar and Zeng [11] investigate a portfolio optimization problem under SV models. Moreover, Ferland and Watier [12] consider a mean-variance portfolio optimization problem with the CIR interest rate in a continuous-time framework, and they derive the mean-variance efficient portfolio by solving backward stochastic differential equations. Li \& Wu [13] and Noh \& Kim [14] consider portfolio optimization problems with an SV asset price process and a stochastic interest rate to maximize the expected utility of the terminal wealth. Li et al. [15] consider the optimal investment and reinsurance problem under Heston's SV model. In addition, [16], [17] and [18] consider the uncertain portfolio selection.

However, in the literature above-mentioned, the past history information of risky asset price is not considered. That is, the price process of risky asset is supposed to follow a geometric Brownian motion with constant drift and constant/stochastic volatility, the future movement of risky asset price is only based on the current information and is independent of the past historic information. However, there is growing evidence to demonstrate that the past price of risky asset influence its future price (See Akgiray [19], Dibeh [20], and Sheinkman \& LeBaron [21]). In other word, investors tend to make their investment decision based on the historic performance of risky asset or their portfolios in the real finance world. Specifically, if a stock price increases a lot recently, then there may be more investors would like to invest more money in this stock, which will push the stock price even higher. On the contrary, if the stock price decreases greatly, more investors tend to sell the stock and invest in other assets, which will drive the price to go down further. The dependence of asset price on the past states is called delay mathematically and a stochastic differential delay equation (SDDE) gives a mathematical formulation for such phenomena. Elsanousi and Larssen [22] investigate a class of optimal consumption problems where the wealth is given by a stochastic differential delay equation with a parameter, and they obtain the closed-form expressions for the optimal strategies and the value functions in two cases of deterministic parameters and random parameters. Chang et al. [23] study an investment and consumption problem of Merton's type modeled by a stochastic system with delay, and they derive the closed-form expressions for the optimal strategies and the value functions in some situations by adopting stochastic control theory. Moreover, Mao [24] studies delay geometric Brownian motion in financial option valuation. Lee et al. [25] study a delayed geometric Brownian model with a stochastic volatility by the martingale method, they extend the geometric Brownian model by adding a stochastic volatility term, which is driven by a hidden process of fast mean reverting diffusion, to the delayed Black-Scholes model. A \& Li [26] consider the optimal 
excess-loss-of reinsurance and investment problem with delay under the Heston's SV model. Shen [27] study the mean-variance portfolio selection in a random environment with unbounded coefficients.

To our best knowledge, there is little work in the literature on portfolio optimization problem when some delay factors (e.g. (2.3)-(2.4) in this paper) are added to the CIR stochastic volatility model. In this paper, we consider a new revised portfolio optimization problem in which we formulate the wealth dynamic as a stochastic differential delay equation with volatility driven by CIR model. By applying the stochastic dynamic programming approach, the corresponding HJB equation and a verification theorem are provided. The closed-form expressions for optimal strategy and optimal value function for CRRA utility model are derived.

The rest of this paper is organized as follows. In Section 2, the model and assumptions are described. In Section 3, the rigorous mathematical formulation of our problem is presented. HJB equation is given, and the verification theorem is proved. The closed-form expressions of optimal strategy and optimal value function for CRRA utility model are derived. In Section 4, some numerical experiment is presented to show our results. Section 5 concludes this paper and states some prospects.

\section{Problem Formulation}

Let $(\Omega, \mathscr{T}, \mathbb{P})$ be a probability space equipped with a filtration $\mathbb{F}=\left(\mathscr{F}_{t}\right)_{0 \leq t \leq T}$ satisfying the usual conditions, i.e., $\left(\mathscr{F}_{t}\right)_{0 \leq t \leq T}$ is right-continuous and $\mathbb{P}$-complete, where $T$ is a positive finite constant representing the time horizon. And all stochastic processes introduced below are supposed to be well-defined and adapted processes in the filtered complete probability space $(\Omega, \mathscr{F}, \mathbb{F}, \mathbb{P})$. In addition, there are no transaction costs or taxes in the financial market and trading takes place continuously.

Consider a financial market consisting of one risk-free asset and one risky asset. The risk-free asset, e.g., a bank account or a bond, can achieve a constant interest rate $r$. The price of risky asset $S_{t}$ is described by following stochastic volatility model, i.e.,

$$
\frac{\mathrm{d} S_{t}}{S_{t}}=\left[\left(r+\eta V_{t}\right)\right] \mathrm{d} t+\sqrt{V_{t}} \mathrm{~d} B_{t}^{S},
$$

where $\eta$ are real constants, $\left\{B_{t}^{S}, t \geq 0\right\}$ is a one-dimension standard Brownian motion; $V_{t}$ is the time varying instantaneous standard deviation of the return on the risk asset. We assume that the instantaneous variance $V_{t}$ follows the CIR process:

$$
\mathrm{d} V_{t}=\kappa\left(\theta-V_{t}\right) \mathrm{d} t+\sigma \sqrt{V_{t}}\left(\rho \mathrm{d} B_{t}^{S}+\sqrt{1-\rho^{2}} \mathrm{~d} B_{t}^{V}\right),
$$

where $\left\{B_{t}^{V}, t \geq 0\right\}$ is a one-dimension Brownian motion defined on the filtered probability space $(\Omega, \mathscr{F}, \mathbb{F}, \mathbb{P})$. Parameter $\theta>0$ describes the long-term mean of the variance, $\kappa \in(0,1)$ is the reversion parameter of the instantaneous va- 
riance process, i.e., $\kappa$ describes the degree of mean reversion, and $\rho$ is the correlation coefficient between Brownian motions $B_{t}^{S}$ and $B_{t}^{V}$, which is assumed to be negative to capture the asymmetric effect.

\subsection{Investment Strategy and Wealth Processes}

Starting from an initial wealth $x_{0}$, an investor invests her wealth in the financial market. Suppose that the investor invests $L_{t}$ and $K_{t}$ dollar in the risk-free asset and the risky asset at time $t$, respectively. Then $X_{t}=L_{t}+K_{t}$ denotes the total wealth at time $t$. In addition, the investor is free to transfer money from the risk-free asset account to the risky asset account and conversely. Let $\left|I_{t}\right|$ be the total dollar amount transferred from one asset to the other asset up to time $t(t \geq 0)$, that is, $I_{t} \geq 0$ means to transfer $I_{t}$ dollar from the risk-free asset account to the risky asset account, $I_{t}<0$ means to transfer $-I_{t}$ dollar from the risky asset account to the risk-free asset account. In addition, define two delay variables by

$$
\begin{gathered}
Y_{t}=\int_{-\delta}^{0} \mathrm{e}^{\lambda s} X(t+s) \mathrm{d} s, \\
Z_{t}=X(t-\delta), \quad \forall t \in[0, T],
\end{gathered}
$$

where $\lambda \geq 0$ is a constant, $\delta>0$ is a delay parameter. From a view of economic point, delay variable $Y_{t}$ and $Z_{t}$ reflect the average and pointwise performance information of the wealth process in the past period $[t-\delta, t]$, respectively. With slight abuse of notation, we do not distinguish $X_{t}$ and $X(t)$. The same is true for other variables.

$L_{t}$ changes with the risk-free interest rate $r$, the dynamic of $L_{t}$ is described by

$$
\mathrm{d} L_{t}=\left(r L_{t}-I_{t}\right) \mathrm{d} t .
$$

Generally, $K_{t}$ changes with risky asset's price. In addition, the historic performance affects the investor's investment decision, further, $K_{t}$ is affected by the historic performance, so we formulate the dynamic of $K_{t}$ as following stochastic differential equation:

$$
\mathrm{d} K_{t}=\left[K_{t}\left(r+v Y_{t}+\mu Z_{t}+\eta V_{t}\right)+I_{t}\right] \mathrm{d} t+\sqrt{V_{t}} K_{t} \mathrm{~d} B_{t}^{S},
$$

where $v, \mu, \eta$ are real constants, $\left\{B_{t}^{S}, t \geq 0\right\}$ is a one-dimension standard Brownian motion, $Y_{t}$ and $Z_{t}$ are given by (2.3) and (2.4). $V_{t}$ satisfies (2.2).

During the investment time horizon $[0, T]$, the investor continuously invests her wealth in the risk-free asset and the risky asset. Let $\pi_{t}$ be the proportion of the investor's wealth invested in the risky asset at time $t$. The remaining proportion $1-\pi_{t}$ is invested in the risk-free asset. Then $K_{t}=\pi_{t} X_{t}$ and $L_{t}=X_{t}-K_{t}=\left(1-\pi_{t}\right) X_{t}$. The process $\pi=\left\{\pi_{t}\right\}_{t[0, T]}$ is called an investment strategy. We assume that short-selling and borrowing are prohibited, i.e., $0 \leq \pi_{t} \leq 1$, and the investment strategies satisfy the self-financial condition, that is, $\mathrm{d} X_{t}=\mathrm{d} K_{t}+\mathrm{d} L_{t}$. Then the dynamic of the wealth $\left\{X_{t}, t \geq 0\right\}$ under the investment strategy $\left\{\pi_{t}\right\}_{t \in[0, T)}$ is given by the following stochastic differential 
delay equation (SDDE):

$$
\mathrm{d} X_{t}=X_{t}\left[\left(\pi_{t}\left(v Y_{t}+\mu Z_{t}+\eta V_{t}\right)+r\right) \mathrm{d} t+\pi_{t} \sqrt{V_{t}} \mathrm{~d} B_{t}^{S}\right], \quad \forall t \in[0, T] .
$$

where $Y_{t}$ and $Z_{t}$ are given by (2.3)-(2.4) and $V_{t}$ satisfies (2.2), respectively. We further assume that $X_{t}=x_{0}>0, t \in[-\delta, 0]$, which can be interpreted that the investor is endowed with the initial wealth $x_{0}$ at time $-\delta$ and do not start investment until time 0 . Then the initial value of the delay variable $Y_{t}$ is

$$
y_{0}:=Y_{0}=\frac{x_{0}\left(1-\mathrm{e}^{-\lambda \delta}\right)}{\lambda} .
$$

Definition 2.1. (ADMISSIBLE STRATEGY) For any fixed $t \in[0, T]$, a strategy $\pi_{t}$ is said to be admissible if it satisfies the following conditions.

i) $\pi_{t}$ is $\mathcal{F}_{t}$-measurable for any $t \in[0, T]$,

ii) For any $t \in[0, T],\left|\pi_{t} X_{t}\right| \leq k\left|X_{t}+\mu Y_{t}\right|$, here $k \geq 0$ is a constant.

Let $\Pi$ denote the set of all admissible strategies.

\subsection{Portfolio Optimization Problem}

In this subsection, we formulate the portfolio optimization problem with delay.

Definition 2.2. A utility function $U:[0, \infty) \times[0, \infty) \rightarrow R\{\infty\}$ is a two variables function i.e. $u=U(x, y),(x, y) \in \mathbb{R}^{2} . u$ is strictly increasing, strictly concave, twice continuously differentiable with respect to the first variable $x$, and satisfies $\lim _{x \rightarrow \infty} u_{x}(x, y)=0$ and $\lim _{x \rightarrow 0} u_{x}(x, y)=\infty$.

We consider an optimization problem of the investor who starts with an initial wealth $x_{0}$ and initial historic information of $y_{0}$ and $z_{0}$. The investor wants to select a investment strategy $\pi \in \Pi$ so as to maximize the expected utility $\mathbb{E}\left[U\left(X_{T}, Y_{T}\right)\right]$. Here, we consider an expected utility of a combination of the terminal wealth $X_{T}$ and the average performance information of the wealth process in the past period $[T-\delta, t]$, i.e., $Y_{T}$. That is, $U\left(X_{T}, Y_{T}\right)$ is the terminal utility function which depends on both the terminal wealth $X_{T}$ and delay variable $Y_{T}$ such that

$$
\mathbb{E}^{t, x, y, v}\left[U\left(X_{T}, Y_{T}\right)\right]<\infty,
$$

for all $x, y$ and $v$, where $\mathbb{E}^{t, x, y, v}[\cdot]=\mathbb{E}\left[\cdot \mid X_{t}=x, Y_{t}=y, V_{t}=v\right]$.

In mathematical terms, the portfolio optimization problem on a finite time horizon $[0, T]$ can be modeled as the following optimization problem.

Problem 2.3. (Portfolio optimization problem with delay)

$$
\begin{gathered}
\sup _{\pi \in \Pi} \mathbb{E}\left[U\left(X_{T}, Y_{T}\right)\right] \\
\text { s.t. } \mathrm{d} X_{t}=X_{t}\left[\left(\pi_{t}\left(v Y_{t}+\mu Z_{t}+\eta V_{t}\right)+r\right) \mathrm{d} t+\pi_{t} \sqrt{V_{t}} \mathrm{~d} Z_{t}^{S}\right], \\
\mathrm{d} V_{t}=\kappa\left(\theta-V_{t}\right) \mathrm{d} t+\sigma \sqrt{V_{t}}\left(\rho \mathrm{d} Z_{t}^{S}+\sqrt{1-\rho^{2}} \mathrm{~d} Z_{t}^{V}\right) .
\end{gathered}
$$

For convenience, we first provide a notation. Let $A_{0} \subset R^{3}$ be an open set and $A=[0, T] \times A_{0}$. Denote 
$C^{1,2,1,2}(A)=\{\phi(t, x, y, z) \mid \phi(t,,, \cdot, \cdot)$ and $\phi(\cdot, \cdot,, z)$ are once continuously differentiable on $[0, T]$ and $\phi(\cdot, x, \cdot, \cdot)$ and $\phi(t, \cdot, y, \cdot$,$\left.) are twice continuously differentiable on A_{0}\right\}$.

First, we show an important Itô's formula. Let $f \in C^{1,2,1,2}\left(\mathbb{R}^{4}\right)$ and define $G(t)=f(t, x, y, v)$,

where

$$
\begin{aligned}
& x=x\left(X_{t}\right), y=y\left(X_{t}\right)=\int_{-\delta}^{0} \mathrm{e}^{\lambda s} X(t+s) \mathrm{d} s, \\
& z=z\left(X_{t}\right)=X(t-\delta), v=V_{t}, \pi=\pi(t) .
\end{aligned}
$$

Lemma 2.4. (Itô's formula)

$$
\begin{aligned}
\mathrm{d} G(t)= & \mathscr{L}_{\pi} f \mathrm{~d} t+f_{x} \pi x \sqrt{v} \mathrm{~d} B_{t}^{S}+f_{v} \sigma \sqrt{v}\left(\rho \mathrm{d} B_{t}^{S}+\sqrt{1-\rho^{2}} \mathrm{~d} B_{t}^{v}\right) \\
& +f_{y}\left[x-\mathrm{e}^{-\lambda \delta} z-\lambda y\right] \mathrm{d} t,
\end{aligned}
$$

where

$$
\begin{aligned}
\mathscr{L}_{\pi} f= & \mathscr{L}_{\pi} f(t, x, y, v) \\
= & f_{t}+x[\pi(v y+\mu z+\eta v)+r] f_{x}+\kappa(\theta-v) f_{v} \\
& +\frac{1}{2} \pi^{2} x^{2} v f_{x x}+\pi \sigma \rho x v f_{v x}+\frac{1}{2} \sigma^{2} v f_{v v} .
\end{aligned}
$$

Proof. For each $t \in[0, T]$, using the Leibnitz formula, by (2.3)-(2.4) we have

$$
\begin{aligned}
\frac{\mathrm{d}}{\mathrm{d} t}\left(y\left(X_{t}\right)\right) & =\frac{\mathrm{d}}{\mathrm{d} t}\left[\int_{-\delta}^{0} \mathrm{e}^{\lambda s} X(t+s) \mathrm{d} s\right] \\
& =\frac{\mathrm{d}}{\mathrm{d} t}\left[\int_{t-\delta}^{t} \mathrm{e}^{\lambda(u-t)} X(u) \mathrm{d} u\right] \quad(u=t+s) \\
& =\zeta(t)-\mathrm{e}^{-\lambda \delta} X(t-h)-\lambda \int_{t-\delta}^{t} \mathrm{e}^{\lambda(u-t)} X(u) \mathrm{d} u \\
& =x\left(\zeta_{t}\right)-\mathrm{e}^{-\lambda \delta} Z\left(X_{t}\right)-\lambda y\left(X_{t}\right) .
\end{aligned}
$$

Since $G(t)=f(t, x, y, v)$, by the classical Itô's formula we can obtain

$$
\begin{aligned}
\mathrm{d} G(t)= & \left\{f_{t}+x[\pi(v y+\mu z+\eta v)+r] f_{x}+\kappa(\theta-v) f_{v}+\frac{1}{2} \pi^{2} x^{2} v f_{x x}\right. \\
& \left.+\pi \sigma \rho x v f_{v x}+\frac{1}{2} \sigma^{2} v f_{v v}\right\} \mathrm{d} t+f_{x} \pi x \sqrt{v} \mathrm{~d} B_{t}^{S} \\
& +f_{v} \sigma \sqrt{v}\left(\rho \mathrm{d} B_{t}^{S}+\sqrt{1-\rho^{2}} \mathrm{~d} B_{t}^{V}\right)+f_{y}\left[x-\mathrm{e}^{-\lambda \delta} z-\lambda y\right] \mathrm{d} t \\
\equiv & \mathscr{L}_{\pi} f \mathrm{~d} t+f_{x} \pi x \sqrt{v} \mathrm{~d} B_{t}^{S}+f_{v} \sigma \sqrt{v}\left(\rho \mathrm{d} B_{t}^{S}+\sqrt{1-\rho^{2}} \mathrm{~d} B_{t}^{V}\right) \\
& +f_{y}\left[x-\mathrm{e}^{-\lambda \delta} z-\lambda y\right] \mathrm{d} t .
\end{aligned}
$$

where $\mathscr{L}_{\pi} f$ is the form of (2.12).

Remark 2.5. Lemma 2.4 yields the following useful formula for $\mathrm{d} y$ :

$$
\mathrm{d} y=\left(x-\mathrm{e}^{-\lambda \delta} z-\lambda y\right) \mathrm{d} t .
$$

\section{Optimal Investment Strategy}

In this section, the HJB equation and a verification of Problem 2.3 are showed. Moreover, the closed-form expression of optimal investment strategy and value 
function are derived for CRRA utility function. By applying the dynamic programming approach, the portfolio optimization problem is equivalent to the problem of finding a solution to the HJB equation.

\subsection{HJB Equation and Verification Theorem}

For an admissible strategy $\pi$, define the value function

$$
J(t, x, y, v)=\mathbb{E}\left[U\left(X_{T}, Y_{T}\right) \mid X_{t}=x, Y_{t}=y, V_{t}=v\right] .
$$

Then the optimal value function is

$$
J^{\pi}(t, x, y, v)=\sup _{\pi \in \Pi} J(t, x, y, v),
$$

with boundary condition $J^{\pi}(T, x, y, v)=U(x, y)$.

Using the Itô's formula in Lemma 2.4, we can show the following HJB equation. Assume that $J(t, x, y, v) \in C^{1,2,1,2}([0, T] \times \mathbb{R} \times \mathbb{R} \times \mathbb{R})$, then the value function $J(t, x, y, v)$ solves the following $\mathrm{HJB}$ equation

$$
\sup _{\pi \in \Pi}\left\{\mathscr{L}_{\pi} J(t, x, y, v)+J_{y}\left(x-\mathrm{e}^{-\lambda \delta} z-\lambda y\right)\right\}=0
$$

with boundary condition

$$
J(T, x, y, v)=U(x, y),
$$

where

$$
\begin{aligned}
\mathscr{L}_{\pi} J(t, x, y, v)= & J_{t}+x[\pi(v y+\mu z+\eta v)+r] J_{x}+\kappa(\theta-v) J_{v} \\
& +\frac{1}{2} \pi^{2} x^{2} v J_{x x}+\pi \sigma \rho x v J_{v x}+\frac{1}{2} \sigma^{2} v J_{v v} .
\end{aligned}
$$

Though we have known that the value function $J(t, x, y, v)$ is the solution of the HJB equation, we need to prove a verification theorem to ensure that a solution to the HJB equation is actually equal to the value function.

Theorem 3.1. (Verification Theorem) Let $X_{t}$ be a strong solution of (2.7), $Y_{t}$ and $Z_{t}$ are given by (2.3) and (2.4), and

$J(t, x, y, v) \in C^{1,2,1,2}([0, T] \times \mathbb{R} \times \mathbb{R} \times \mathbb{R})$ is a solution of HJB Equation (3.3) with boundary condition (3.4) such that

$$
\mathbb{E}\left\{\int_{0}^{T}\left(\sqrt{v} J_{v}\right)^{2} \mathrm{~d} t\right\}<\infty, \quad \mathbb{E}\left\{\int_{0}^{T}\left(\pi_{t} \sqrt{v} x J_{x}\right)^{2} \mathrm{~d} t\right\}<\infty, \quad \forall \pi \in \Pi .
$$

Then we have

$$
J(t, x, y, v) \geq \sup _{\pi \in \Pi} \mathbb{E}^{t, x, y, v}\left[U\left(X_{T}, Y_{T}\right)\right]
$$

Further let

$$
\pi^{*}=-\frac{(v y+\mu z+\eta v) J_{x}+\sigma \rho v J_{x v}}{x v J_{x v}} .
$$

If $\pi^{*} \in \Pi$, then $\pi^{*}$ is the optimal strategy of Problem 2.3 and

$$
J(t, x, y, v)=\mathbb{E}^{t, x, y, v}\left[U\left(X_{T}, Y_{T}\right) \mid \pi=\pi^{*}\right] .
$$

Proof. Let $J(t, x, y, v)$ be a solution of the HJB equation (3.3) with boundary 
condition (3.4). For any given admissible strategy $\pi \in \Pi$ and for any $(t, x, y, v) \in[0, T] \times \mathbb{R} \times \mathbb{R} \times \mathbb{R}$, we must have

$$
\left\{\mathscr{L}_{\pi} J(t, x, y, v)+J_{y}\left(x-\mathrm{e}^{-\lambda \delta} z-\lambda y\right)\right\} \leq 0 .
$$

On the other hand, applying Itô's formula (2.11) to $J(t, x, y, v)$, we have

$$
\begin{aligned}
\mathrm{d}[J(t, x, y, v)]= & {\left[\mathscr{L}_{\pi} J(t, x, y, v)+J_{y}\left(x-\mathrm{e}^{-\lambda \delta} z-\lambda y\right)\right] \mathrm{d} t } \\
& +\pi_{t} \sqrt{v} x J_{x} \mathrm{~d} B_{t}^{S}+f_{v} \sigma \sqrt{v}\left(\rho \mathrm{d} B_{t}^{S}+\sqrt{1-\rho^{2}} \mathrm{~d} B_{t}^{V}\right) .
\end{aligned}
$$

Integrating it from $s$ to $T$, and using (3.8), we obtain

$$
\begin{aligned}
J & \left(T, X_{T}, Y_{T}, V_{T}\right)-J\left(s, X_{s}, Y_{s}, V_{s}\right) \\
= & \int_{s}^{T}\left\{\mathscr{L}_{\pi} J(t, x, y, v)+J_{y}\left(x-\mathrm{e}^{-\lambda \delta} z-\lambda y\right)\right\} \mathrm{d} t \\
& +\int_{s}^{T} \pi_{t} \sqrt{v} x J_{x} \mathrm{~d} B_{t}^{S}+\int_{s}^{T} f_{v} \sigma \sqrt{v}\left(\rho \mathrm{d} B_{t}^{S}+\sqrt{1-\rho^{2}} \mathrm{~d} B_{t}^{V}\right) \\
\leq & \int_{s}^{T} \pi_{t} \sqrt{v} x J_{x} \mathrm{~d} B_{t}^{S}+\int_{s}^{T} f_{v} \sigma \sqrt{v}\left(\rho \mathrm{d} B_{t}^{S}+\sqrt{1-\rho^{2}} \mathrm{~d} B_{t}^{V}\right) .
\end{aligned}
$$

Therefore, by virtue of boundary condition (3.4), we have

$$
J\left(s, X_{s}, Y_{s}, V_{s}\right) \geq U\left(X_{T}, Y_{T}\right)-\int_{s}^{T} \pi_{t} \sqrt{v} x J_{x} \mathrm{~d} B_{t}^{S}-\int_{S}^{T} f_{v} \sigma \sqrt{v}\left(\rho \mathrm{d} B_{t}^{S}+\sqrt{1-\rho^{2}} \mathrm{~d} B_{t}^{V}\right) .
$$

Using the condition (3.6), it is easy to see that

$$
\int_{s}^{T} f_{v} \sqrt{v} \mathrm{~d} B_{t}^{S}, \int_{s}^{T} f_{v} \sqrt{v} \mathrm{~d} B_{t}^{V} \text { and } \int_{s}^{T} \pi_{t} \sqrt{v} x J_{x} \mathrm{~d} B_{t}^{S}
$$

are square integrable martingale whose expectation vanish. Therefore, by taking expectations on both sides of (3.9), we derive

$$
J(t, x, y, v) \geq \mathbb{E}^{t, x, y, v}\left[U\left(X_{T}, Y_{T}\right)\right] .
$$

Because it holds for all $\pi \in \Pi$, we must get

$$
J(t, x, y, v) \geq \sup _{\pi \in \Pi} \mathbb{E}^{t, x, y, v}\left[U\left(X_{T}, Y_{T}\right)\right] .
$$

On the other hand, if we take $\pi=\pi^{*}$ as defined in (3.7), and if $\pi^{*} \in \Pi$, then all the above inequalities can be replaced by equalities. In other words, we have

$$
J(t, x, y, v)=\mathbb{E}^{t, x, y, v}\left[U\left(X_{T}, Y_{T}\right) \mid \pi=\pi^{*}\right] .
$$

Now the proof is complete.

\subsection{Solution of HJB Equation}

To obtain a closed-form solution of HJB equation (3.3) with boundary condition (3.4), we assume that the investor has a utility function of the following form

$$
U(x, y)=\frac{1}{1-\gamma}\left(x+\mu \mathrm{e}^{\lambda \delta} y\right)^{1-\gamma},
$$

where $\gamma>0, \gamma \neq 1$, and $\gamma$ is the investor's relative risk aversion coefficient. In fact, Chang et al. [23] and Elsanousi [22] also adopt the utility function of (3.10) to study the optimal investment and consumption problem with delay.

The maximum in HJB Equation (3.3) is obtained when 


$$
\frac{\partial}{\partial \pi}\left(\mathscr{L}_{\pi} J(t, x, y, v)+J_{Y}\left(x-\mathrm{e}^{-\lambda \delta} z-\lambda y\right)\right)=0,
$$

furthermore,

$$
\pi^{*}=-\frac{(v y+\mu z+\eta v) J_{x}+\sigma \rho v J_{v x}}{v x J_{x x}} .
$$

We try to seek a function $J$ satisfying the HJB equation (3.3) in the form

$$
J(t, x, y, v)=\frac{\left(x+\mu \mathrm{e}^{\lambda \delta} y\right)^{1-\gamma}}{1-\gamma} \exp \{\gamma h(T-t)+\gamma H(T-t) v\} \text {. }
$$

Define

$$
u \equiv x+\mu \mathrm{e}^{\lambda \delta} y, \quad \tilde{H}(t)=\exp \{\gamma h(T-t)+\gamma H(T-t) v\}
$$

It is easy to verify that

$$
\begin{aligned}
& J_{t}=\frac{u^{1-\gamma}}{1-\gamma}\left(-\gamma h^{\prime}-\gamma H^{\prime} v\right) \tilde{H}(t), \quad J_{y}=\mu \mathrm{e}^{\lambda \delta} \tilde{H}(t) u^{-\gamma}, \quad J_{x}=\tilde{H}(t) u^{-\gamma}, \\
& J_{v}=\frac{\gamma}{1-\gamma} H \tilde{H}(t) u^{1-\gamma}, \quad J_{x x}=(-\gamma) \tilde{H}(t) u^{-\gamma-1}, \quad J_{x v}=\gamma H \tilde{H}(t) u^{-\gamma}, \\
& J_{v v}=\frac{u^{1-\gamma}}{1-\gamma}(\gamma H)^{2} \tilde{H}(t) .
\end{aligned}
$$

Plugging them into (3.11), we can derive

$$
\pi^{*}=\frac{(v y+\mu z+\eta v+\gamma \sigma \rho v H) u}{\gamma v x} .
$$

Substituting (3.13)-(3.14) into (3.3) yields

$$
\begin{aligned}
\frac{\gamma}{1-\gamma}\left(h^{\prime}+v H^{\prime}\right) u^{1-\gamma}= & x\left((v y+\mu z+\eta v) \frac{(v y+\mu z+\eta v+\gamma \sigma \rho v H) u}{\gamma v x}+r\right) u^{-\gamma} \\
& +u^{-\gamma} \mu \mathrm{e}^{\lambda \delta}\left(x-\mathrm{e}^{-\lambda \delta} z-\lambda y\right)+\frac{u^{1-\gamma}}{1-\gamma} \gamma \kappa(\theta-v) H \\
& +\frac{1}{2} u^{-\gamma-1} \frac{(v y+\mu z+\eta v+\gamma \sigma \rho v H)^{2} u^{2}}{\gamma v} \\
& +u^{-\gamma} \gamma \sigma \rho v x H \frac{(v y+\mu z+\eta v+\gamma \sigma \rho v H) u}{\gamma v x} \\
& +\frac{1}{2} \frac{u^{1-\gamma}}{1-\gamma} \sigma^{2} \gamma^{2} v H^{2} .
\end{aligned}
$$

Let $Q=v y+\eta v$. From (3.15) we have

$$
\begin{aligned}
\frac{\gamma}{1-\gamma}\left(h^{\prime}+v H^{\prime}\right) u^{1-\gamma}= & \frac{(Q+\mu z)(Q+\gamma \sigma \rho v H+\mu z)}{\gamma v} u^{1-\gamma}+r x u^{-\gamma}+\mu \mathrm{e}^{\lambda \delta}(x-\lambda y) u^{-\gamma} \\
& -\mu z u^{-\gamma}+\frac{\gamma}{1-\gamma} \kappa(\theta-v) H u^{1-\gamma}-\frac{1}{2} \frac{(Q+\gamma \sigma \rho v H+\mu z)^{2}}{\gamma v} u^{1-\gamma} \\
& +\frac{1}{2} \frac{\gamma^{2} \sigma^{2} v H^{2}}{1-\gamma} u^{1-\gamma}+(Q+\mu z+\gamma \sigma \rho v H) \rho \sigma H u^{1-\gamma},
\end{aligned}
$$

or equivalently, 


$$
\begin{aligned}
\frac{\gamma}{1-\gamma}\left(h^{\prime}+v H^{\prime}\right) u^{1-\gamma}= & \frac{Q(Q+\gamma \sigma \rho v H)}{\gamma V} u^{1-\gamma}+\frac{\gamma}{1-\gamma} \kappa(\theta-v) H u^{1-\gamma} \\
& +\frac{1}{2} \frac{\gamma^{2} \sigma^{2} v H^{2}}{1-\gamma} u^{1-\gamma}+(Q+\gamma \sigma \rho v H) \rho \sigma H u^{1-\gamma} \\
& +\left[\left(r+\mu \mathrm{e}^{\lambda \delta}\right) x-\lambda \mu \mathrm{e}^{\lambda \delta} y\right] u^{-\gamma}-\frac{1}{2} \frac{(Q+\gamma \sigma \rho v H)^{2}}{\gamma v} u^{1-\gamma} \\
& +\left(\frac{\mu z Q}{\gamma v}+\frac{1}{2} \frac{\mu^{2} z^{2}}{\gamma v}+\mu \rho \sigma z H\right) u^{1-\gamma}-\mu z u^{-\gamma} .
\end{aligned}
$$

Since (3.12) has a solution that does not depend on $Z$, we have the following condition

$$
\left(\frac{\mu z Q}{\gamma v}+\frac{1}{2} \frac{\mu^{2} z^{2}}{\gamma v}+\mu \rho \sigma z H\right) u^{1-\gamma}-\mu z u^{-\gamma}=0 .
$$

By (3.18), (3.17) becomes

$$
\begin{aligned}
\frac{\gamma}{1-\gamma}\left(h^{\prime}+v H^{\prime}\right) u^{1-\gamma}= & \frac{Q(Q+\gamma \sigma \rho v H)}{\gamma v} u^{1-\gamma}+\frac{\gamma}{1-\gamma} \kappa(\theta-v) H u^{1-\gamma} \\
& +\frac{1}{2} \frac{\gamma^{2} \sigma^{2} v H^{2}}{1-\gamma} u^{1-\gamma}+(Q+\gamma \sigma \rho v H) \rho \sigma H u^{1-\gamma} \\
& -\frac{1}{2} \frac{(Q+\gamma \sigma \rho v H)^{2}}{\gamma v} u^{1-\gamma}+\left[\left(r+\mu \mathrm{e}^{\lambda \delta}\right) x-\lambda \mu \mathrm{e}^{\lambda \delta} y\right] u^{-\gamma} .
\end{aligned}
$$

Equation (3.17) has a solution depending only on $t$ and $u$ if

$$
-\lambda=\mu \mathrm{e}^{\lambda \delta}+r, \quad \lambda<0 .
$$

Plugging $Q$ and (3.19) into (3.17), we have

$$
\begin{aligned}
h^{\prime}+v H^{\prime}= & \frac{1-\gamma}{\gamma}\left(\frac{v^{2} y^{2}}{2 \gamma v}+\frac{v \eta y}{\gamma}+v \rho \sigma y H\right)+\left(\kappa \theta H+\frac{1-\gamma}{\gamma}\left(r+\mu \mathrm{e}^{\lambda \delta}\right)\right) \\
& +\left(\frac{1}{2} \sigma^{2}\left(\gamma\left(1-\rho^{2}\right)+\rho^{2}\right) H^{2}+\left[\frac{(1-\gamma) \eta \rho \sigma}{\gamma}-\kappa\right] H+\frac{1-\gamma}{2 \gamma^{2}} \eta^{2}\right) v .
\end{aligned}
$$

Equation (3.20) is equivalent to the following ordinary differential equations

$$
\left\{\begin{array}{l}
\frac{v^{2} y^{2}}{2 \gamma \nu}+\frac{v \eta y}{\gamma}+v \rho \sigma y H=0 \\
h^{\prime}=\kappa \theta H+\frac{1-\gamma}{\gamma}\left(r+\mu \mathrm{e}^{\lambda \delta}\right) \\
H^{\prime}=\frac{1}{2} \sigma^{2}\left(\gamma\left(1-\rho^{2}\right)+\rho^{2}\right) H^{2}+\left[\frac{(1-\gamma) \eta \rho \sigma}{\gamma}-\kappa\right] H+\frac{1-\gamma}{2 \gamma^{2}} \eta^{2}
\end{array}\right.
$$

From boundary condition (3.4), we have

$$
H(T)=0, \quad h(T)=0 .
$$

Solving equations (3.21) with boundary condition (3.22), we derive the following Theorem.

Theorem 3.2. (Optimal strategy of Problem 2.3) Given wealth $X_{t}$ and CRRA utility (3.10), the solution to HJB Equation (3.3) is given by 


$$
J\left(t, X_{t}, Y_{t}, V_{t}\right)=\frac{1}{1-\gamma}\left(X_{t}+\mu \mathrm{e}^{\lambda \delta} Y_{t}\right)^{1-\gamma} \exp \left\{\gamma h(T-t)+\gamma H(T-t) V_{t}\right\},
$$

where $h(\cdot)$ and $H(\cdot)$ are time-dependent coefficients that are independent of the state variables. That is, for any $0 \leq \tau \leq T$,

$$
\begin{gathered}
H(\tau)=\frac{\exp \left(k_{3} \tau\right)-1}{2 k_{3}+\left(k_{1}+k_{3}\right)\left(\exp \left(k_{3} \tau\right)-1\right)} \vartheta, \\
h(\tau)=\frac{2 \kappa \theta}{\sigma^{2}} \ln \left(\frac{2 k_{3} \exp \left(\left(k_{1}+k_{3}\right) \tau / 2\right)}{2 k_{3}+\left(k_{1}+k_{3}\right)\left(\exp \left(k_{3} \tau\right)-1\right)}\right)+\frac{1-\gamma}{\gamma}(r+\mu \exp (\lambda \delta)) \tau,
\end{gathered}
$$

where

$$
\vartheta=\frac{1-\gamma}{\gamma^{2}} \eta^{2}, k_{1}=\kappa-\frac{1-\gamma}{\gamma} \eta \rho \sigma, k_{2}=\sigma^{2}\left(\gamma\left(1-\rho^{2}\right)+\rho^{2}\right), k_{3}=\sqrt{k_{1}^{2}-\vartheta k_{2}} .
$$

The optimal investment proportion in the risky asset of Problem 2.3 is given by

$$
\pi_{t}^{*}=\frac{\left(v Y_{t}+\mu Z_{t}+\eta V_{t}+\gamma \sigma \rho V_{t} H(T-t)\right)\left(X_{t}+\mu \mathrm{e}^{\lambda \delta} Y_{t}\right)}{\gamma V_{t} X_{t}} .
$$

Remark 3.3. It is interesting that our results are similar to the results of Liu and Pan [28]. Liu and Pan study optimal investment strategies given an investor access not only to bond and stock markets but also to the derivatives market and the price process of the risk asset is associated with stochastic volatility and jump. Here are some comparisons between them.

i) In our results, the proportion in the risky asset depends on wealth $X_{t}$, delay variables $Y_{t}$ and $Z_{t}$, and stochastic volatility $V_{t}$ at time $t$. However, in Liu and Pan [28] the optimal strategy is a deterministic function and does not depend on wealth $X_{t}$ and stochastic volatility $V_{t}$. Moreover, our results are consistent with the results of Liu and Pan [28], when the delay variables are not considered in our model (i.e. $v=\mu=0$ ). The details are given in Lemma 3.4.

ii) Let the delay $\delta$ approach 0 then $Y_{t} \rightarrow 0$, in this case the delay variable $Y_{t}$ vanishes. Assuming that (3.19) holds and $\lambda=-r$, then $\mu=0$. At this time, the dynamics of wealth (2.7) degenerates as

$$
\mathrm{d} X_{t}=X_{t}\left[\left(\pi_{t} \eta V_{t}+r\right) \mathrm{d} t+\pi_{t} \sqrt{V_{t}} \mathrm{~d} Z_{t}^{S}\right],
$$

which is the case without delay. The corresponding problem without delay and its optimal strategy are given in Lemma 3.4.

iii) The value function $J\left(t, X_{t}, Y_{t}, V_{t}\right)$ of (3.23) depends only on delay variable $Y_{t}$ and does not depend on delay variable $Z_{t}$.

\subsection{A Special Case}

Now we consider a special case of our model. Suppose that the dynamics of risky asset does not depend on historic performance, then our model degenerates into a CIR SV model without delay. The results of our model will be reduced to the following special case. 
Proposition 3.4. (Optimal strategy without delay) Consider the following problem without delay:

$$
\begin{gathered}
\max _{\pi_{t} \in \Pi} \mathbb{E}\left[U\left(X_{T}\right)\right]=\mathbb{E}\left[\frac{X_{T}^{1-\gamma}}{1-\gamma}\right] \\
\text { s.t. } \mathrm{d} X_{t}=X_{t}\left[\left(\pi_{t} \eta V_{t}+r\right) \mathrm{d} t+\pi_{t} \sqrt{V_{t}} \mathrm{~d} B_{t}^{S}\right], \\
\mathrm{d} V_{t}=\kappa\left(\theta-V_{t}\right) \mathrm{d} t+\sigma \sqrt{V_{t}}\left(\rho \mathrm{d} B_{t}^{S}+\sqrt{1-\rho^{2}} \mathrm{~d} B_{t}^{V}\right) .
\end{gathered}
$$

The value function is given by

$$
J(t, x, v)=\max _{\pi_{t} \in \Pi} \mathbb{E}\left[U\left(X_{T}\right) \mid X_{t}=x, V_{t}=v\right]
$$

and $\mathrm{HJB}$ equation is given by

$\max \left\{J_{t}+x(\pi \eta v+r) J_{x}+\kappa(\theta-v) J_{v}+\frac{1}{2} \pi^{2} x^{2} v J_{x x}+\pi \sigma \rho v x J_{v x}+\frac{1}{2} \sigma^{2} v J_{v v}\right\}=0$

with boundary condition $J(T, x, v)=\frac{x^{1-\gamma}}{1-\gamma}$.

Then, for given wealth $X_{t}$, the solution to HJB equation is given by

$$
J\left(t, X_{t}, V_{t}\right)=\frac{1}{1-\gamma} X_{t}^{1-\gamma} \exp \left\{\gamma h(T-t)+\gamma H(T-t) V_{t}\right\}
$$

where $h(\cdot)$ and $H(\cdot)$ are time-dependent coefficients that are independent of the state variables. That is, for any $0 \leq \tau \leq T$,

$$
\begin{gathered}
H(\tau)=\frac{\exp \left(k_{3} \tau\right)-1}{2 k_{3}+\left(k_{1}+k_{3}\right)\left(\exp \left(k_{3} \tau\right)-1\right)} \vartheta, \\
h(\tau)=\frac{2 \kappa \theta}{\sigma^{2}} \ln \left(\frac{2 k_{3} \exp \left(\left(k_{1}+k_{3}\right) \tau / 2\right)}{2 k_{3}+\left(k_{1}+k_{3}\right)\left(\exp \left(k_{3} \tau\right)-1\right)}\right)+\frac{1-\gamma}{\gamma} r \tau,
\end{gathered}
$$

where

$$
\vartheta=\frac{1-\gamma}{\gamma^{2}} \eta^{2}, k_{1}=\kappa-\frac{1-\gamma}{\gamma} \eta \rho \sigma, k_{2}=\sigma^{2}\left(\gamma\left(1-\rho^{2}\right)+\rho^{2}\right), k_{3}=\sqrt{k_{1}^{2}-\vartheta k_{2}} .
$$

The optimal investment proportion in the risky asset is given by

$$
\bar{\pi}_{t}^{*}=\frac{\eta+\gamma \sigma \rho H(T-t)}{\gamma}
$$

Proof. Let $\mu=v=0$ in Theorem 4.5, we can easily obtain the results of this lemma.

Remark 3.5. Our results without considering delay (i.e. $v=\mu=0$ ) is similar to the results of Liu and Pan [28]. Their results are consistent with our results without considering delay, when there is only stock and not derivatives considering in Liu and Pan's model. In some sense, we extend the results of Liu and Pan [28].

\section{Numerical Experiment}

In this section, we investigate the effect of delay variables, stochastic volatility 
and VaR constraint on the optimal strategies and the optimal value functions, and provide some numerical examples to demonstrate the effect. We set the initial wealth level in million dollars between 0 and 10. The VaR horizon period is chosen to be 1 trading day, nearly $1 / 360$ calendar year, while the terminal year is set to be 10 calendar years. In the following numerical illustrations, unless otherwise stated, the basic parameters are given by $r=0.05, \alpha=0.01, \eta=2, \rho=-0.9, \sigma=0.1, \kappa=0.2, \delta=1$.

\subsection{Analysis of Optimal Strategy}

In general, the dynamic changes of wealth $X_{t}$ must depend on both delay variables $Y_{t}$ and $Z_{t}$ at the same time in a similar manner, i.e., $v \mu \geq 0$.

(1) From (3.27), for given $X>0$ and $V>0$, we have

$$
\begin{gathered}
\frac{\partial \pi^{*}}{\partial Y}=\frac{v X+\mu \mathrm{e}^{\lambda \delta}}{\gamma V X} \begin{cases}=0, & v=\mu=0, \\
>0, & v>0, \mu>0 \\
<0, & v<0, \mu<0,\end{cases} \\
\frac{\partial \pi^{*}}{\partial Z}=\frac{\mu}{\gamma V X} \begin{cases}=0, & \mu=0, \\
>0, & \mu>0, \\
<0, & \mu<0,\end{cases}
\end{gathered}
$$

According to the above results, we know that i) if $v=\mu=0$, then $\frac{\partial \pi^{*}}{\partial Y}=\frac{\partial \pi^{*}}{\partial Z}=0$, which is the case without delay, the optimal investment strategy $\pi^{*}$ dose not depend on $Y$ and $Z$; ii) if $v<0$ and $\mu<0$, then $\frac{\partial \pi^{*}}{\partial Y}>0$ and $\frac{\partial \pi^{*}}{\partial Z}>0$, which mean that the delay factors take a positive effect on the optimal investment strategy; iii) if $v<0$ and $\mu<0$, then $\frac{\partial \pi^{*}}{\partial Y}<0$ and $\frac{\partial \pi^{*}}{\partial Z}<0$, which mean that the delay factors take a passive effect on the optimal investment strategy.

In Figure 1, we draw the optimal investment strategy $\pi^{*}$ with $t$ under the different delay. Let $v=\mu=0$ (or $Y=0, Z=0$ ) denote the case without delay, i.e., the curves denoted by " $Y=0$ " in two Figures. In Figure 1(a), we set $v=0.01, \mu=0.01, Z=2$, and $Y=2$ or $Y=20$ respectively. Here, $v=0.01>0$ and $\mu=0.01>0$ mean that delay variables $Y$ and $Z$ take a positive effect on optimal investment strategy, that is, the curves are arranged by $Y=20, Y=2$ and $Y=0$ from top to bottom. In other words, the higher positive historic performance of the portfolio promote the higher proportion of an investor's wealth invested on stocks. From another point of view, the result implies that a higher historic stock price encourages investor to invest more money to the stock. Conversely, in Figure 1(b), let $v=-0.01, \mu=-0.01, Z=2$, and $Y=2$ or $Y=20$ respectively. And $v=0.01<0$ and $\mu=0.01<0$ mean that delay variables $Y$ and $Z$ take a negative effect on optimal investment strategy, that is, the curves are arranged by $Y=0, Y=2$ and $Y=20$ from top to bottom. 


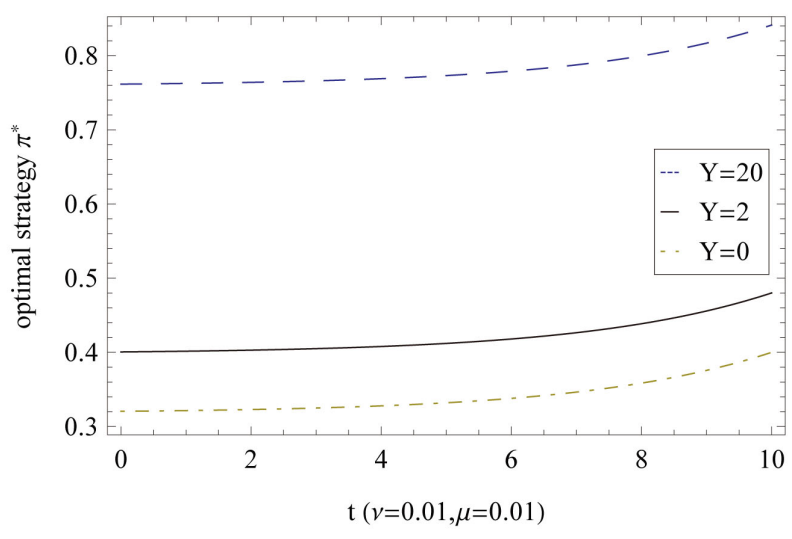

(a)

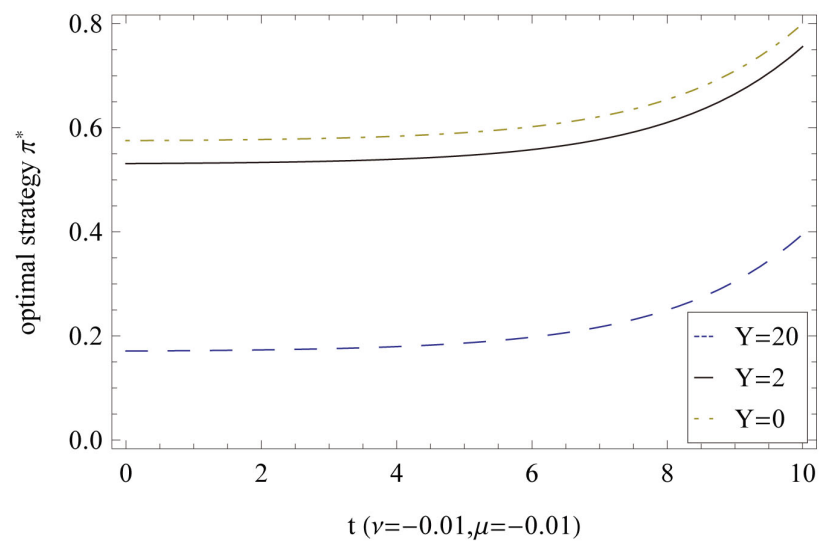

(b)

Figure 1. Optimal strategies with time $t$ under different delay.

Figure 1(b) shows that the higher historic performance of the portfolio gives the investors a chance to harvest their gain by cut down the proportion invested on stocks, which implies that a higher historic stock price cause investor to invest less money on the stock so as to avoid to chase the high price. This seems to be consistent with the facts.

(2) From (3.27), for given $Y>0$ and $Z>0$, we have

$$
\begin{aligned}
& \frac{\partial \pi^{*}}{\partial V}=-\frac{(v Y+\mu Z)\left(X+\mu \mathrm{e}^{\lambda \delta}\right)}{\gamma X V^{2}}<0, \\
& \frac{\partial \pi^{*}}{\partial X}=-\frac{\nu Y+\mu Z+\eta V+\gamma \sigma \rho H(T-\tau) \mu \mathrm{e}^{\lambda \delta}}{\gamma V}<0 .
\end{aligned}
$$

So, the optimal investment strategy $\pi^{*}$ decrease w.r.t. stochastic volatility $V$ and wealth $X$, respectively.

Figure 2 plot the optimal investment strategy $\pi^{*}$ with stochastic volatility $V$ under the different delay. Let $X=100, \gamma=10 . v=\mu=0$ or $Y=0, Z=0$ means the case without delay, i.e., the curve denoted by " $Y=0$ " in two Figures. Figure 2 shows that the optimal investment strategy $\pi^{*}$ decreases with increasing stochastic volatility $V$. That is to say, the higher stochastic volatility take a passive effect on the investment enthusiasm. Moreover, in Figure 2(a), we set $v=0.01, \mu=0.01, Z=2$, and $Y=2$ or $Y=20$ respectively. Here,

$v=0.01>0$ and $\mu=0.01>0$ mean that delay variables $Y$ and $Z$ take a positive effect on optimal strategy, that is, the curves are arranged by $Y=20, Y=2$ and $Y=0$ from top to bottom. Conversely, in Figure 2(b), let $v=-0.01, \mu=-0.01, Z=2$, and $Y=2$ or $Y=20$ respectively. $v=0.01<0$ and $\mu=0.01<0$ mean that delay variables $Y$ and $Z$ take a negative effect on optimal investment strategy, that is, the curves are arranged by $Y=0, Y=2$ and $Y=20$ from top to bottom. That is to say, Figure 1 and Figure 2 have the same result about the effect of delay variables on the optimal investment strategy.

In Figure 3, we demonstrates the optimal investment strategy $\pi^{*}$ w.r.t. 


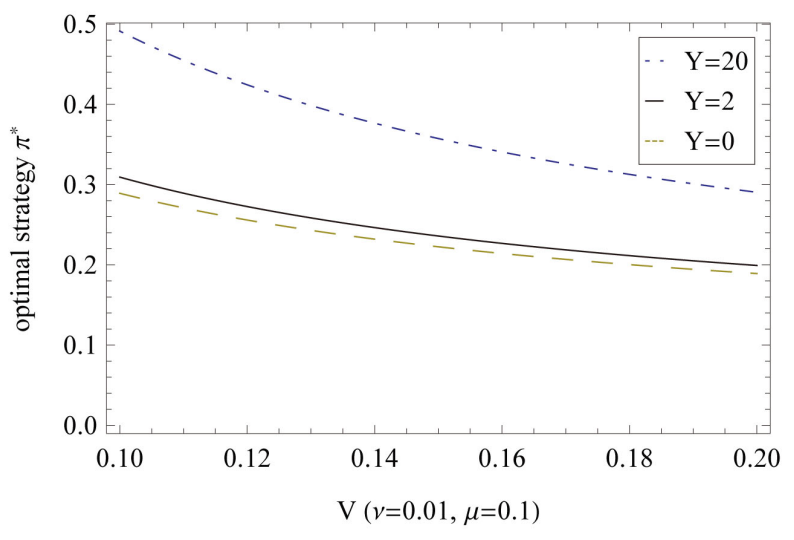

(a)

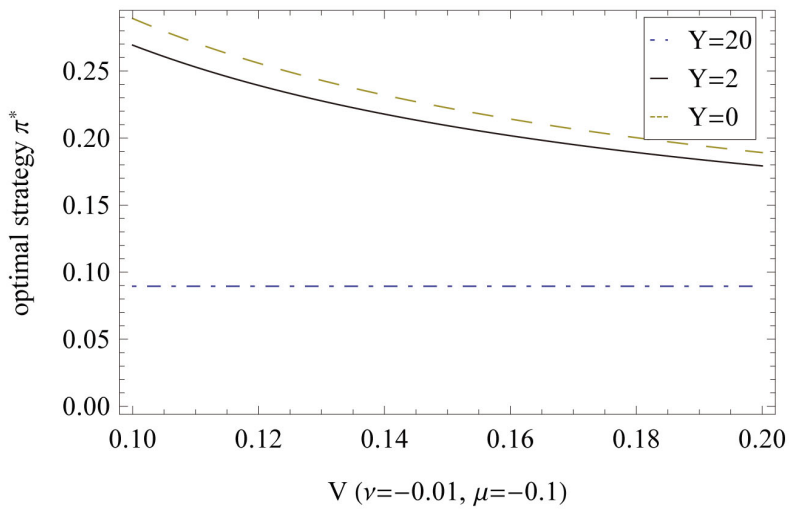

(b)

Figure 2. Optimal strategies with stochastic volatility V under different delay.

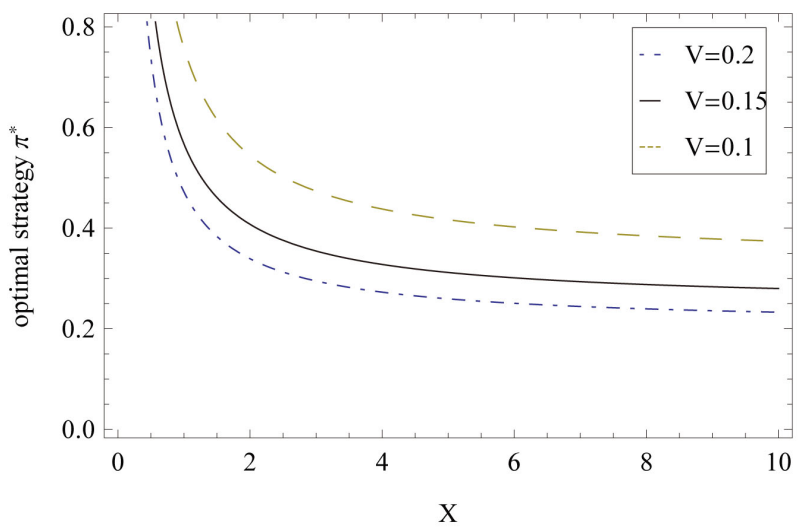

(a)

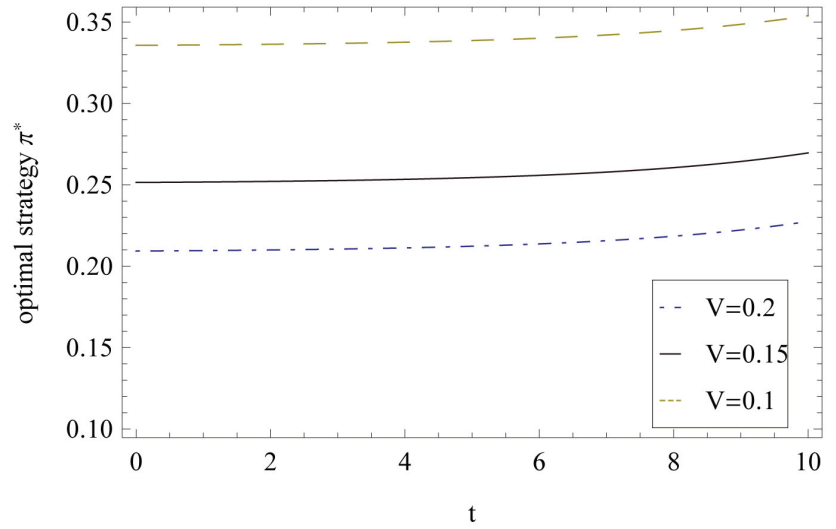

(b)

Figure 3. Optimal strategies under the different stochastic volatility.

different wealth $X$ under the different volatility $V$. Let $V=0.1, V=0.15$, and $V=0.2$ respectively, and with delay variables $Y=2, Z=2, \mu=0.01, v=0.1$. The curves are arranged by $V=0.1, V=0.15$, and $V=0.2$ from top to bottom. The two figure show the larger is stochastic volatility $V$ and the smaller is the proportion of the investor's wealth invested on stocks. This fits with the fact that market instability has negative impact on investors. Furthermore, in Figure 3(a) the curves show that the optimal investment strategy $\pi^{*}$ decreases with the accumulation of wealth $X$. This suggests that an investor has an increasing preference to risk aversion with the increase of wealth, thereby, an investor cut down the stockholding.

\subsection{Analysis of Optimal Value Function}

In this subsection, we analyze the effect of delay variable $Y$ and stochastic volatility $V$ on the value function. According to (3.23), the value function depends on $t, X, Y, V$ and we have

$$
J_{X}=\left(X+\mu \mathrm{e}^{\lambda \delta} Y\right)^{-\gamma} \exp \{\gamma h(T-t)+\gamma H(T-t) V\}>0,
$$




$$
\begin{gathered}
J_{V}=\frac{\left(X+\mu \mathrm{e}^{\lambda \delta} Y\right)^{1-\gamma}}{1-\gamma} \gamma H(T-t) \exp \{\gamma h(T-t)+\gamma H(T-t) V\}>0, \\
J_{Y}=\mu \mathrm{e}^{\lambda \delta}\left(X+\mu \mathrm{e}^{\lambda \delta} Y\right)^{-\gamma}>0 \quad(\mu>0),
\end{gathered}
$$

Let $X=100, v=0.01, \mu=0.01, Z=2, Y=2$ or $Y=200$, and $v=\mu=0 \quad$ (or $Y=0, Z=0$ ) denotes without delay in Figure 5 and Figure 6 (i.e., the curve denoted by " $Y=0$ ").

Figure 4 plot the optimal value function $J(t, X, Y, V)$ with $t$ when the risk aversion coefficient $\gamma=0.3$ and $\gamma=3$. They shows that the value functions are almost the same with different delay variables.

Figure 5 plot the optimal value function $J(t, X, Y, V)$ with wealth $X$ when the risk aversion coefficient $\gamma=0.3$ and $\gamma=3$. They shows that the optimal value functions are almost the same with different delay variables. Figure 6 plot the optimal value function $J(t, X, Y, V)$ w.r.t. stochastic volatility $V$ when the risk aversion coefficient $\gamma=0.3$ and $\gamma=3$. However, it is interesting enough that the higher stochastic volatility seemed to induce the higher value of the value functions. That is, the curves are arranged by $V=0.1, V=0.15$, and $V=0.2$ from top to bottom. The two figures show that the optimal value functions increase with the increasing of stochastic volatility $V$.

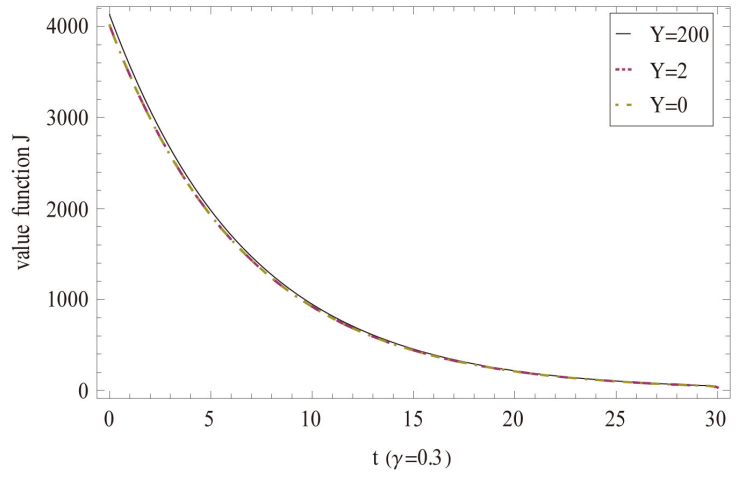

(a)

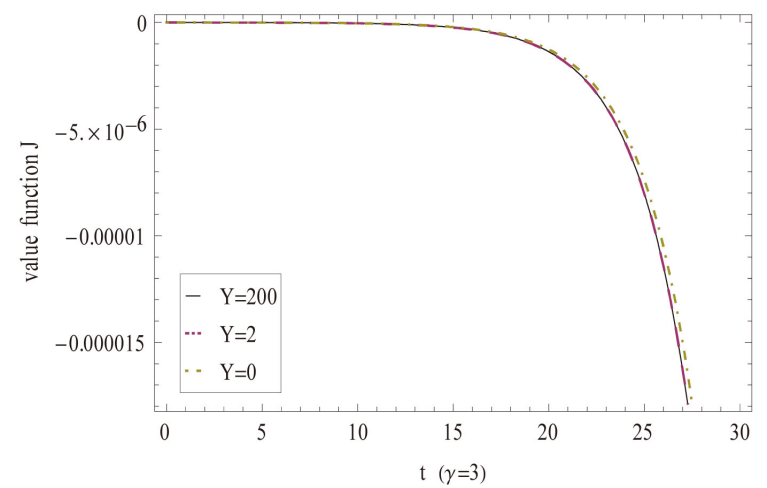

(b)

Figure 4. Optimal value function w.r.t time $t$ under the different delay.

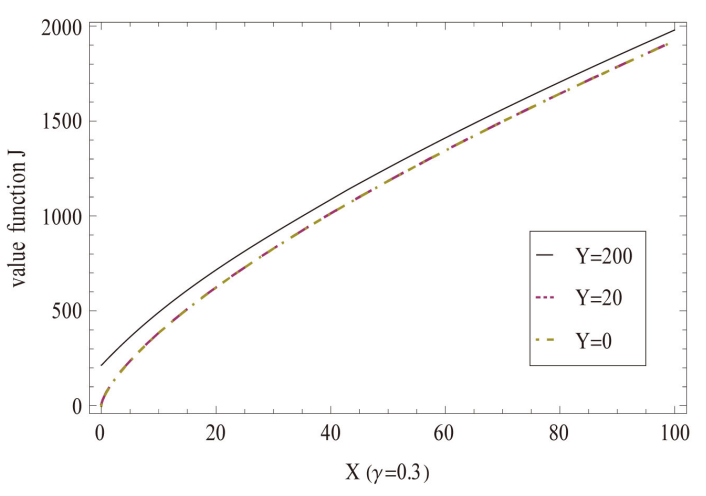

(a)

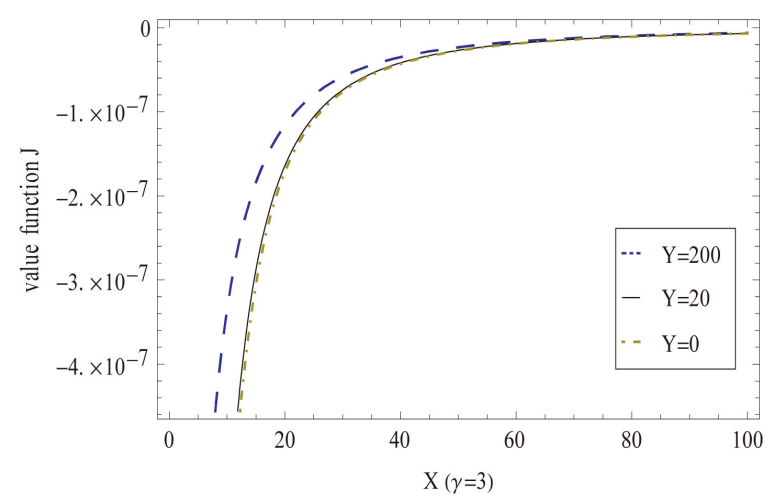

(b)

Figure 5. Optimal value function w.r.t. wealth $X$ under the different delay. 


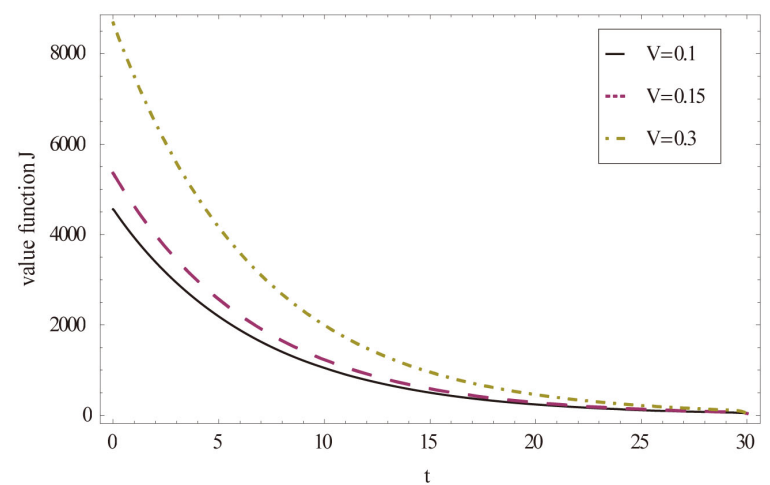

(a)

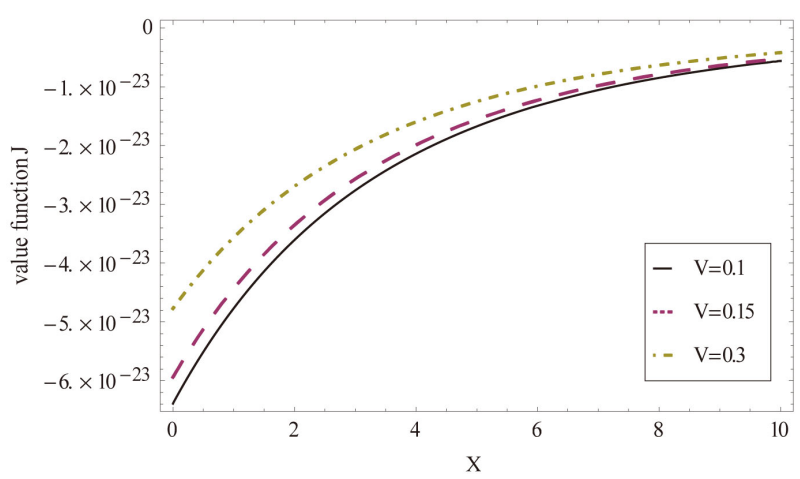

(b)

Figure 6. Optimal value function under different stochastic volatility $V$.

\section{Conclusions and Prospect}

This paper considers portfolio optimization problem with delay under CIR stochastic volatility model. Adopting the stochastic dynamic programming approach, we derive the optimal portfolio strategy in closed-form for a CRRA type utility function, and verification theorem is showed.

The results show that the historic performance of portfolio has obvious effect on the optimal strategy. Specifically, the higher positive history performance seems to induce the higher investment proportion on risky asset. On the contrary, the higher negative historic performance of the portfolio leads to the lower proportion on risky asset. And the historic performance of portfolio has similar effect on value function. As a result, it is meaningful to put delay variables into the portfolio optimization problem.

There are several topics which deserve to be studied in the future. First, as illustrated in this paper, the portfolio optimization problem with a single risky and single risk-free asset obtains an explicit solution via the dynamic programming principle and the verification theorem. However, it is anticipated that explicit solutions of the similar type for the model with multiple risky assets will not be available.

\section{Supported}

This research is supported by NSFC (No. 71501050) and Startup Foundation for Doctors of ZhaoQing University (No. 611-612282).

\section{References}

[1] Hull, J. and White, A. (1987) The Pricing of Options on Assets with Stochastic Volatilities. Journal of Finance, 42, 281-300. https://doi.org/10.1111/j.1540-6261.1987.tb02568.x

[2] Scott, L.O. (1987) Option Pricing When the Variance Changes Randomly Theory Estimation, and an Application. Journal of Financial and Quantitative Analysis, 22, 419-438. https://doi.org/10.2307/2330793

[3] Stein, E.M. and Stein, J.C. (1991) Stock Price Distributions with Stochastic Volatility: An Analytic Approach. Review of Financial Studies, 4, 727-752. 
https://doi.org/10.1093/rfs/4.4.727

[4] Heston, S.L. (1993) A Closed-Form Solution for Options with Stochastic Volatility with Applications to Bond and Currency Options. Review of Financial Studies, 6, 327-343. https://doi.org/10.1093/rfs/6.2.327

[5] Ball, C.A. and Roma, A. (1994) Stochastic Volatility Option Pricing. Journal of Financial and Quantitative Analysis, 29, 589-607. https://doi.org/10.2307/2331111

[6] Chacko, G. and Viceira, L.M. (2005) Dynamic Consumption and Portfolio Choice with Stochastic Volatility in Incomplete Markets. Review of Financial Studies, 8, 1369-1402. https://doi.org/10.1093/rfs/hhi035

[7] Fleming, W.H. and Hernandez-Hernandez, D. (2003) An Optimal Consumption Model with Stochastic Volatility. Finance and Stochastics, 7, 245-262. https://doi.org/10.1007/s007800200083

[8] Liu, J. (2007) Portfolio Selection in Stochastic Environment. Review of Financial Studies, 20, 1-39. https://doi.org/10.1093/rfs/hhl001

[9] Zariphopoulou, T. (1999) Optimal Investment and Consumption Models with Nonlinear Stock Dynamics. Mathematical Methods of Operations Research, 50, 271-296. https://doi.org/10.1007/s001860050098

[10] Kraft, H. (2005) Optimal Portfolio and Heston's Stochastic Volatility Model: An Explicit Solution for Power Utility. Quantitative Finance, 5, 303-313. https://doi.org/10.1080/14697680500149503

[11] Taksar, M.I. and Zeng, X.D. (2009). A General Stochastic Volatility Model and Optimal Portfolio with Explicit Solutions. Working Paper. http://www.math.missouri.edu/zeng/pub/ageneral.pdf.

[12] Ferland, R. and Watier, F. (2010) Mean-Variance Efficiency with Extended CIR Interest Rates. Applied Stochastic Models in Business and Industry, 26, 71-84. https://doi.org/10.1002/asmb.767

[13] Li, J.Z. and Wu, R. (2009) Optimal Investment Problem with Stochastic Interest Rate and Stochastic Volatility: Maximizing a Power Utility. Applied Stochastic Models in Business and Industry, 25, 407-420. https://doi.org/10.1002/asmb.759

[14] Noh, E.J. and Kim, J.H. (2010) An Optimal Portfolio Model with Stochastic Volatility and Stochastic Interest Rate. Journal of Mathematical Analysis and Applications, 375, 510-522. https://doi.org/10.1016/j.jmaa.2010.09.055

[15] Li, Z.F., Zeng, Y. and Lai, Y.Z. (2012) Optimal Time-Consistent Investment and Reinsurance Strategies for Insurers under Heston's SV Model. Insurance: Mathematics and Economics, 51, 191-203.

https://doi.org/10.1016/j.insmatheco.2011.09.002

[16] Chen, L., Peng, J., Zhang, B. and Rosyida, I. (2017) Diversified Models for Portfolio Selection Based on Uncertain Semivariance. International Journal of Systems Science, 48, 637-648. https://doi.org/10.1080/00207721.2016.1206985

[17] Qin, Z.F., Kar, S. and Zheng, H.T. (2016) Uncertain Portfolio Adjusting Model Using Semiabsolute Deviation. Soft Computing, 20, 717-725. https://doi.org/10.1007/s00500-014-1535-y

[18] Zhang, B., Peng, J. and Li, S.G. (2015) Uncertain Programming Models for Portfolio Selection with Uncertain Returns. International Journal of Systems Science, 46, 2510-2519. https://doi.org/10.1080/00207721.2013.871366

[19] Akgiray, V. (1998) Conditional Heteroscedasticity in Time Series of Stock Returns. Journal of Business, 62, 55-80. https://doi.org/10.1086/296451

[20] Dibeh, G. (2005) Speculative Dynamics in a Time-Delay Model of Asset Prices. Physica A, 355, 199-208. https://doi.org/10.1016/j.physa.2005.02.084 
[21] Sheinkman, J. and LeBaron, B. (1989) Nonlinear Dynamics and Stock Returns. Journal of Business, 62, 311-337. https://doi.org/10.1086/296465

[22] Elsanousi, L. and Larssen, B. (2001) Optimal Consumption under Partial Observations for a Stochastic System with Delay. Preprint 9, University of Oslo, Oslo.

[23] Chang, M.-H., Pang, T. and Yang, Y.P. (2011) A Stochastic Portfolio Optimization Model with Bounded Memory. Mathematics of Operations Research, 36, 604-619. https://doi.org/10.1287/moor.1110.0508

[24] Mao, X.R. (2012) Delay Geometric Brownian Motion in Financial Option Valuation. Stochastics, 1-26.

[25] Lee, M.K., Kim, J.H. and Kim, J. (2011) A Delay Financial Model with Stochastic Volatility: Martingale Method. Physica A, 390, 2909-2919. https://doi.org/10.1016/j.physa.2011.03.032

[26] A, C.X. and Li, Z.F. (2015) Optimal Investment and Excess-of-Loss Reinsurance Problem with Delay for an Insurer under Heston's SV Model. Insurance: Mathematics and Economics, 61, 181-196.

https://doi.org/10.1016/j.insmatheco.2015.01.005

[27] Shen, Y. and Zeng, Y. (2014) Optimal Investment-Reinsurance with Delay for Mean-Variance Insurers: A Maximum Principle Approach. Insurance: Mathematics and Economics, 57, 1-12. https://doi.org/10.1016/j.insmatheco.2014.04.004

[28] Liu, J. and Pan, J. (2003) Dynamic Derivative Strategies. Journal of Financial Economics, 69, 401-430. https://doi.org/10.1016/S0304-405X(03)00118-1

Submit or recommend next manuscript to SCIRP and we will provide best service for you:

Accepting pre-submission inquiries through Email, Facebook, LinkedIn, Twitter, etc. A wide selection of journals (inclusive of 9 subjects, more than 200 journals)

Providing 24-hour high-quality service

User-friendly online submission system

Fair and swift peer-review system

Efficient typesetting and proofreading procedure

Display of the result of downloads and visits, as well as the number of cited articles

Maximum dissemination of your research work

Submit your manuscript at: http://papersubmission.scirp.org/

Or contact jmf@scirp.org 